\title{
Hyperspaces of Finite Sets in Universal Spaces for Absolute Borel Classes
}

\author{
by \\ Kotaro MINE, Katsuro SAKAI and Masato YAGUCHI \\ Presented by Czestaw BESSAGA
}

Summary. By $\operatorname{Fin}(X)$ (resp. $\left.\operatorname{Fin}^{k}(X)\right)$, we denote the hyperspace of all non-empty finite subsets of $X$ (resp. consisting of at most $k$ points) with the Vietoris topology. Let $\ell_{2}(\tau)$ be the Hilbert space with weight $\tau$ and $\ell_{2}^{\mathrm{f}}(\tau)$ the linear span of the canonical orthonormal basis of $\ell_{2}(\tau)$. It is shown that if $E=\ell_{2}^{\mathrm{f}}(\tau)$ or $E$ is an absorbing set in $\ell_{2}(\tau)$ for one of the absolute Borel classes $\mathfrak{a}_{\alpha}(\tau)$ and $\mathfrak{M}_{\alpha}(\tau)$ of weight $\leq \tau(\alpha>0)$ then Fin $(E)$ and each $\operatorname{Fin}^{k}(E)$ are homeomorphic to $E$. More generally, if $X$ is a connected $E$-manifold then $\operatorname{Fin}(X)$ is homeomorphic to $E$ and each $\operatorname{Fin}^{k}(X)$ is a connected $E$-manifold.

1. Introduction. Throughout the paper, spaces are metrizable and maps are continuous. Let $\tau$ be an infinite cardinal. Let $\ell_{2}(\tau)$ be the Hilbert space of weight $\tau$ and $\ell_{2}^{\mathrm{f}}(\tau)$ the linear span of the canonical orthonormal basis of $\ell_{2}(\tau)$. We write $\ell_{2}\left(\aleph_{0}\right)=\ell_{2}$ and $\ell_{2}^{\mathrm{f}}\left(\aleph_{0}\right)=\ell_{2}^{\mathrm{f}}$. Given a space $E$, a paracompact Hausdorff space is called a manifold modeled on $E$ or an $E$-manifold if it can be covered by open sets which are homeomorphic to $(\approx)$ open sets in $E$.

In [1], Bestvina and Mogilski constructed universal spaces for separable absolute Borel classes as absorbing sets in the Hilbert cube $Q=\mathbf{I}^{\mathbb{N}}$ or the pseudo-interior $s=(0,1)^{\mathbb{N}}$, and they also gave topological characterizations of those spaces and manifolds modeled on them. Recently, in [14] and [8], the present authors generalized the results of [1] to non-separable absolute Borel classes. For each countable ordinal $\alpha>0$, let $\Lambda_{\alpha}(\tau)$ and $\Omega_{\alpha}(\tau)$ be absorbing sets in $\ell_{2}(\tau)$ for the classes $\mathfrak{a}_{\alpha}(\tau)$ and $\mathfrak{M}_{\alpha}(\tau)$, respectively, where $\mathfrak{a}_{\alpha}(\tau)$ and $\mathfrak{M}_{\alpha}(\tau)$ are respectively the additive and multiplicative absolute

2000 Mathematics Subject Classification: 54B20, 57N20, 54H05.

Key words and phrases: hyperspace of finite subsets, Vietoris topology, Hausdorff metric, universal spaces, absolute Borel classes, Hilbert space, absorbing set. 
Borel classes $\alpha$ with weight $\leq \tau$. Then $\Lambda_{\alpha}(\tau)(\alpha \geq 1)$ and $\Omega_{\alpha}(\tau)(\alpha \geq 2)$ are universal spaces in the classes $\mathfrak{a}_{\alpha}(\tau)$ and $\mathfrak{M}_{\alpha}(\tau)$, respectively. The space $\Omega_{1}(\tau)$ is homeomorphic to $\ell_{2}(\tau) \times \ell_{2}^{\mathrm{f}}$, which is a universal space in the class of $\sigma$-completely metrizable spaces $\left({ }^{1}\right)$ with weight $\leq \tau$. Note that this class is a proper subclass of $\mathfrak{a}_{2}(\tau)$. Although $\mathfrak{a}_{1}\left(\aleph_{0}\right)$ is the class of $\sigma$-compact metrizable spaces, $\mathfrak{a}_{1}(\tau)$ is in general the class of $\sigma$-locally compact metrizable $\left({ }^{2}\right)$ spaces with weight $\leq \tau$ (cf. [15]). Moreover, $\Lambda_{1}(\tau) \approx \ell_{2}^{\mathrm{f}}(\tau) \times Q$ and $\Omega_{2}(\tau) \approx \Omega_{1}(\tau)^{\mathbb{N}} \approx \Lambda_{1}(\tau)^{\mathbb{N}}$ (see [8]).

On the other hand, in [3], Curtis and Nguyen To Nhu proved that

the hyperspace $\operatorname{Fin}(X)$ of non-empty finite subsets of $X$ with the Vietoris topology is homeomorphic to $\ell_{2}^{\mathrm{f}}$ if and only if $X$ is nondegenerate, connected, locally path-connected $\sigma$-compact and strongly countable-dimensional.

Moreover, Curtis [2] showed that

$$
\operatorname{Fin}(Q) \approx \operatorname{Fin}\left(\ell_{2}^{\mathrm{f}} \times Q\right) \approx \ell_{2}^{\mathrm{f}} \times Q .
$$

Recently, the last author [19] proved that

$$
\operatorname{Fin}\left(\ell_{2}(\tau)\right) \approx \ell_{2}(\tau) \times \ell_{2}^{\mathrm{f}}\left(\approx \Omega_{1}(\tau)\right) .
$$

For each $k \in \mathbb{N}$, let $\operatorname{Fin}^{k}(X)$ be the subspace of $\operatorname{Fin}(X)$ consisting of subsets with cardinality $\leq k$. For this hyperspace, the following are known:

(1) $\operatorname{Fin}^{k}(Q) \approx Q$ (Fedorchuk [6]),

(2) $\operatorname{Fin}^{k}\left(\ell_{2}\right) \approx \ell_{2}$ and $\operatorname{Fin}^{k}\left(\ell_{2}^{\mathrm{f}} \times Q\right) \approx \ell_{2}^{\mathrm{f}} \times Q$ (Nguyen To Nhu [10]),

(3) $\operatorname{Fin}^{k}\left(\ell_{2} \times \ell_{2}^{\mathrm{f}}\right) \approx \ell_{2} \times \ell_{2}^{\mathrm{f}}$ and $\operatorname{Fin}^{k}\left(\left(\ell_{2}^{\mathrm{f}}\right)^{\mathbb{N}}\right) \approx\left(\ell_{2}^{\mathrm{f}}\right)^{\mathbb{N}}$ (Nguyen To Nhu and the second author [11]),

where $\ell_{2}^{\mathrm{f}} \times Q \approx \Lambda_{1}\left(\aleph_{0}\right), \ell_{2} \times \ell_{2}^{\mathrm{f}} \approx \Omega_{1}\left(\aleph_{0}\right)$ and $\left(\ell_{2}^{\mathrm{f}}\right)^{\mathbb{N}} \approx \Omega_{2}\left(\aleph_{0}\right)$. In this paper, we show the following theorem:

Main Theorem. Let $E$ be one of the spaces $\ell_{2}^{\mathrm{f}}(\tau), \Lambda_{\alpha}(\tau), \Omega_{\alpha}(\tau)$, where $\alpha>0$. Then $\operatorname{Fin}(E) \approx E$ and $\operatorname{Fin}^{k}(E) \approx E$ for each $k \in \mathbb{N}$. More generally if $X$ is a connected E-manifold then $\operatorname{Fin}(X) \approx E$ and each $\operatorname{Fin}^{k}(X)$ is a connected E-manifold.

2. Preliminaries. For a metrizable space $X$, let $\mathfrak{a}_{0}(X)$ and $\mathfrak{M}_{0}(X)$ be the collections of all open sets and of all closed sets in $X$, respectively. For a countable ordinal $\alpha>0$, by transfinite induction, we define $\mathfrak{a}_{\alpha}(X)$ (resp. $\left.\mathfrak{M}_{\alpha}(X)\right)$ as the collection of all countable unions (resp. intersections) of sets in $\bigcup_{\beta<\alpha} \mathfrak{a}_{\beta}(X) \cup \mathfrak{M}_{\beta}(X)$. Then $\mathfrak{M}_{1}(X)$ and $\mathfrak{a}_{1}(X)$ are the collections of all

$\left({ }^{1}\right)$ A metrizable space is $\sigma$-completely metrizable if it is a countable union of completely metrizable closed subsets.

$\left({ }^{2}\right)$ A metrizable space is $\sigma$-locally compact metrizable if it is a countable union of locally compact (closed) subsets. See footnote 1 in [8]. 
$G_{\delta}$ sets and of all $F_{\sigma}$ sets in $X$, respectively. Sets in $\mathfrak{a}_{2}(X), \mathfrak{M}_{2}(X), \ldots$ are said to be $G_{\delta \sigma}, F_{\sigma \delta}, \ldots$ in $X$, respectively. For each subset $A \subset X$, we have $\operatorname{Fin}(A) \subset \operatorname{Fin}(X)$. It is easy to see that $\operatorname{Fin}(A)$ is open (resp. closed) in $\operatorname{Fin}(X)$ if $A$ is open (resp. closed) in $X$. Then, by transfinite induction on $\alpha$, we have

$$
\begin{gathered}
A \in \mathfrak{a}_{\alpha}(X) \Rightarrow \operatorname{Fin}(A) \in \mathfrak{a}_{\alpha}(\operatorname{Fin}(X)), \\
A \in \mathfrak{M}_{\alpha}(X) \Rightarrow \operatorname{Fin}(A) \in \mathfrak{M}_{\alpha}(\operatorname{Fin}(X)) .
\end{gathered}
$$

For an infinite cardinal $\tau$, we denote by $\mathfrak{a}_{\alpha}(\tau)\left(\operatorname{resp} . \mathfrak{M}_{\alpha}(\tau)\right)$ the class of all metrizable spaces $X$ with $w(X) \leq \tau$ such that $X \in \mathfrak{a}_{\alpha}(Y)$ (resp. $\left.X \in \mathfrak{M}_{\alpha}(Y)\right)$ whenever $X$ is embedded in a metrizable space $Y$. Then $\mathfrak{M}_{1}(\tau)$, $\mathfrak{a}_{1}(\tau), \mathfrak{M}_{2}(\tau), \mathfrak{a}_{2}(\tau), \ldots$ are the classes of absolutely $G_{\delta}$, absolutely $F_{\sigma}$, absolutely $G_{\delta \sigma}$, absolutely $F_{\sigma \delta}, \ldots$ spaces with weight $\leq \tau$. Note that $\mathfrak{a}_{0}(\tau)=\emptyset$ and $\mathfrak{M}_{0}(\tau)=\mathfrak{M}_{0}\left(\aleph_{0}\right)$ is the class of compact metrizable spaces. As is well known, absolutely $G_{\delta}$ spaces are nothing else than completely metrizable spaces. A separable metrizable space is absolutely $F_{\sigma}$ if and only if it is $\sigma$-compact. In the general case, a metrizable space is absolutely $F_{\sigma}$ if and only if it is $\sigma$-locally compact (cf. [15]). We denote by $\mathfrak{a}_{1}^{\omega}(\tau)$ the class of all spaces with weight $\leq \tau$ which are countable unions of locally compact, locally finite-dimensional closed sets.

Let $\mathcal{C}$ be a class of spaces. Then

- $\mathcal{C}$ is topological if $(X \in \mathcal{C}, X \approx Y) \Rightarrow Y \in \mathcal{C}$,

- $\mathcal{C}$ is closed (resp. open) hereditary if $(X \in \mathcal{C}, A \subset X$ is closed (resp. open) in $X) \Rightarrow A \in \mathcal{C}$,

- $\mathcal{C}$ is additive if $\left(X=X_{1} \cup X_{2}\right.$ and $X_{1}, X_{2} \in \mathcal{C}$ are closed in $\left.X\right) \Rightarrow$ $X \in \mathcal{C}$.

- $\mathcal{C}$ is productive if $X_{1}, X_{2} \in \mathcal{C} \Rightarrow X_{1} \times X_{2} \in \mathcal{C}$.

By $\mathcal{C}_{\sigma}$, we denote the class consisting of all metrizable spaces which can be expressed as countable unions of closed subspaces contained in $\mathcal{C}$. Then $\mathfrak{M}_{1}(\tau)_{\sigma}$ is the class of $\sigma$-completely metirzable spaces with weight $\leq \tau$. Clearly, if $\mathcal{C}$ is closed hereditary then $\mathcal{C}_{\sigma}$ is closed and open hereditary.

Now, suppose that $\mathcal{C}$ is the topological class $\mathfrak{a}_{\alpha}(\tau)(\alpha \geq 1), \mathfrak{M}_{\alpha}(\tau)(\alpha \geq 2)$ or $\mathfrak{a}_{1}^{\omega}(\tau)$. Then $\mathcal{C}=\mathcal{C}_{\sigma}$ is open and closed hereditary, additive, productive and contains $\mathbf{I}^{n} \times D(\tau)$ for all $n \in \mathbb{N}$, where $D(\tau)$ is the discrete space with card $D(\tau)=\tau$.

For each space $X$, we denote by $\mathcal{E}(X)$ the class of all spaces which are homeomorphic to a closed subset of $X$. Note that

$$
\begin{gathered}
\mathcal{E}\left(\Lambda_{\alpha}(\tau)\right)=\mathfrak{a}_{\alpha}(\tau) \quad(\alpha \geq 1), \quad \mathcal{E}\left(\Omega_{\alpha}(\tau)\right)=\mathfrak{M}_{\alpha}(\tau) \quad(\alpha \geq 2) \\
\mathcal{E}\left(\Omega_{1}(\tau)\right)=\mathfrak{M}_{1}(\tau)_{\sigma} \quad \text { and } \quad \mathcal{E}\left(\ell_{2}^{\mathrm{f}}(\tau)\right)=\mathfrak{a}_{1}^{\omega}(\tau)
\end{gathered}
$$


For each open cover $\mathcal{U}$ of $Y$, two maps $f, g: X \rightarrow Y$ are $\mathcal{U}$-close (or $f$ is $\mathcal{U}$-close to $g$ ) if each $\{f(x), g(x)\}$ is contained in some $U \in \mathcal{U}$. A closed set $A \subset X$ is called a $Z$-set (resp. a strong $Z$-set) in $X$ provided, for each open cover $\mathcal{U}$ of $X$, there is a map $f: X \rightarrow X$ such that $f$ is $\mathcal{U}$-close to $\operatorname{id}_{X}$ and $f(X) \cap A=\emptyset$ (resp. cl $f(X) \cap A=\emptyset)$. A subset $X \subset Y$ is homotopy dense in $Y$ if there is a homotopy $h: Y \times \mathbf{I} \rightarrow Y$ such that $h_{0}=\operatorname{id}_{Y}$ and $h_{t}(Y) \subset X$ for every $t>0$. When $X$ is an ANR, a closed set $A$ is a $Z$-set in $X$ if and only if $X \backslash A$ is homotopy dense in $X$ (cf. Corollary 3.3 of [16]). A countable union of (strong) $Z$-sets in $X$ is called a (strong) $Z_{\sigma}$-set in $X$. A (strong) $Z_{\sigma}$-space is a (strong) $Z_{\sigma}$-set in itself. A $Z$-embedding is an embedding whose image is a $Z$-set.

A space $X$ is said to be universal for a class $\mathcal{C}$ (simply, $\mathcal{C}$-universal) if every map $f: C \rightarrow X$ of $C \in \mathcal{C}$ can be approximated by $Z$-embeddings. We say that $X$ is strongly universal for $\mathcal{C}$ (simply, strongly $\mathcal{C}$-universal) when the following condition is satisfied:

$\left(\mathrm{su}_{\mathcal{C}}\right) \quad$ for each $C \in \mathcal{C}$ and each closed set $D \subset C$, if $f: C \rightarrow X$ is a map such that $f \mid D$ is a $Z$-embedding, then, for each open cover $\mathcal{U}$ of $X$, there is a $Z$-embedding $h: C \rightarrow X$ such that $h|D=f| D$ and $h$ is $\mathcal{U}$-close to $f$.

A $\mathcal{C}$-absorbing set in $Y$ is a homotopy dense subset $X \subset Y$ such that $X \in \mathcal{C}_{\sigma}$ and $X$ is a strongly $\mathcal{C}$-universal strong $Z_{\sigma}$-space. In [13, Theorem 3.8], Sakai and Yaguchi generalized a characterization of $\mathcal{C}$-absorbing sets by Bestvina and Mogilski [1, Theorem 5.3] to the following non-separable case:

THEOREM 2.1. Let $\mathcal{C}$ be a closed hereditary additive topological class of spaces such that $\mathbf{I}^{n} \times D(\tau) \in \mathcal{C}$ for each $n \in \mathbb{N}$. Suppose that there exists a $\mathcal{C}$-absorbing set $E$ in $\ell_{2}(\tau)$. Then an $A R X$ with $w(X) \leq \tau$ is homeomorphic to $E$ if and only if $X \in \mathcal{C}_{\sigma}, X$ is strongly $\mathcal{C}$-universal and $X$ is a strong $Z_{\sigma}$-space.

The open embedding theorem of $E$-manifolds [1, Corollary 5.7] can also be generalized to the non-separable case [13, Theorem 3.9] as follows:

THEOREM 2.2. Under the assumption of Theorem 2.1, every connected E-manifold can be embedded in $E$ as an open set.

In this paper, $X_{\mathrm{f}}^{\mathbb{N}}$ denotes the weak product of $X$ with a base point $* \in X$, that is,

$$
X_{\mathrm{f}}^{\mathbb{N}}=\left\{\left(x_{i}\right)_{i \in \mathbb{N}} \in X^{\mathbb{N}} \mid x_{i}=* \text { except for finitely many } n \in \mathbb{N}\right\} .
$$

In order to make the base point clear, we write $X_{\mathrm{f}}^{\mathbb{N}}(*)$. As is easily observed, Proposition 2.5 of [1] is valid for a non-separable AR $X$. Then we have the following proposition: 
Proposition 2.3. Under the assumptions of Theorem 2.1, if $\mathcal{C}$ is productive then $E \approx E_{\mathrm{f}}^{\mathbb{N}}$.

Proof. Since $\mathcal{C} \subset \mathcal{E}(E) \subset \mathcal{E}\left(E_{\mathrm{f}}^{\mathbb{N}}\right)$, it follows from Proposition 2.5 of [1] that $E_{\mathrm{f}}^{\mathbb{N}}$ is strongly $\mathcal{E}(E)$-universal. Moreover, $E_{\mathrm{f}}^{\mathbb{N}} \subset \ell_{2}(\tau)^{\mathbb{N}} \approx \ell_{2}(\tau)$ and $E$ is homotopy dense in $\ell_{2}(\tau)$. Then $E_{\mathrm{f}}^{\mathbb{N}}$ can be embedded into $\ell_{2}(\tau)^{\mathbb{N}}$ as a homotopy dense subset. By Lemma 2.2 of [8], $E_{\mathrm{f}}^{\mathbb{N}}$ is a strong $Z_{\sigma^{-}}$space. Since $E \in \mathcal{C}_{\sigma}$ and $\mathcal{C}$ is productive, we have $E_{\mathrm{f}}^{\mathbb{N}} \in \mathcal{C}_{\sigma}$. Hence, $E \approx E_{\mathrm{f}}^{\mathbb{N}}$ by Theorem 2.1.

The following is due to Nguyen To Nhu [9, Theorem 2.1, Corollary 2.3]:

TheOrem 2.4. For every ANR (resp. AR) $X$ and $k \in \mathbb{N}$, the hyperspaces $\operatorname{Fin}(X)$ and $\operatorname{Fin}^{k}(X)$ are also $A N R$ 's (resp. AR's).

Note that every map $f: X \rightarrow Y$ induces a map $\tilde{f}: \operatorname{Fin}(X) \rightarrow \operatorname{Fin}(Y)$ defined by $\tilde{f}(A)=f(A)=\{f(x) \mid x \in A\}$. Moreover, for a homotopy $h: X \times \mathbf{I} \rightarrow Y$, we define $\widetilde{h}: \operatorname{Fin}(X) \times \mathbf{I} \rightarrow \operatorname{Fin}(Y)$ and $\widetilde{h}^{k}: \operatorname{Fin}^{k}(X) \times \mathbf{I} \rightarrow$ $\operatorname{Fin}^{k}(Y)$ for each $k \in \mathbb{N}$ by $\widetilde{h}_{t}(A)=\widetilde{h}_{t}^{k}(A)=h_{t}(A)=\left\{h_{t}(x) \mid x \in A\right\}$. Then it is easy to see that $\widetilde{h}$ and $\widetilde{h}^{k}$ are continuous, so they are also homotopies.

3. Universality. Given an admissible metric $d$ for $X$, we use the admissible metric for $X^{\mathbb{N}}$ defined as follows:

$$
\varrho\left(\left(x_{i}\right)_{i \in \mathbb{N}},\left(y_{i}\right)_{i \in \mathbb{N}}\right)=\sup _{i \in \mathbb{N}} \min \left\{d\left(x_{i}, y_{i}\right), 2^{-i}\right\} .
$$

Then $\varrho_{\mathrm{H}}$ is the Hausdorff metric induced by the metric $\varrho$.

Proposition 3.1. Let $X$ be a non-degenerate $A R$ and $W$ an open set in $\operatorname{Fin}\left(X_{\mathrm{f}}^{\mathbb{N}}\right)$ or $\operatorname{Fin}^{k}\left(X_{\mathrm{f}}^{\mathbb{N}}\right)$ for some $k \in \mathbb{N}$. Then $W$ is universal for $\mathcal{E}(X)$.

Proof. Because of similarity, we shall prove only the case of $\operatorname{Fin}\left(X_{\mathrm{f}}^{\mathbb{N}}\right)$. Let $Y=X_{\mathrm{f}}^{\mathbb{N}} \subset X^{\mathbb{N}}$. Since the weak product $Y_{\mathrm{f}}^{\mathbb{N}}(*)$ of $Y$ with a base point $* \in Y$ is homeomorphic to $X_{\mathrm{f}}^{\mathbb{N}}$, we may show the universality of every open set $W$ in $\operatorname{Fin}\left(Y_{\mathrm{f}}^{\mathbb{N}}(*)\right)$. Let $f: A \rightarrow W$ be a map of $A \in \mathcal{E}(X)$. For each open cover $\mathcal{U}$ of $W$, take a collection $\widetilde{\mathcal{U}}$ of open sets of $\operatorname{Fin}\left(\left(X^{\mathbb{N}}\right)^{\mathbb{N}}\right)$ such that $\mathcal{U}=\left\{U \cap \operatorname{Fin}\left(Y_{\mathrm{f}}^{\mathbb{N}}(*)\right) \mid U \in \widetilde{\mathcal{U}}\right\}$. Then $\widetilde{W}=\bigcup \widetilde{\mathcal{U}}$ is an open subset of $\operatorname{Fin}\left(\left(X^{\mathbb{N}}\right)^{\mathbb{N}}\right)$. Suppose that $\alpha: \widetilde{W} \rightarrow(0,1)$ is a map such that if a map $g: Y \rightarrow \operatorname{Fin}\left(\left(X^{\mathbb{N}}\right)^{\mathbb{N}}\right)$ is $\alpha$-close to $f$ then $g(Y) \subset \widetilde{W}$ and $g$ is $\widetilde{\mathcal{U}}$-close to $f$. Since $Y$ is an AR, we have a map $\lambda: Y \times Y \times \mathbf{I} \rightarrow Y$ such that $\lambda(x, x, t)=x$ for every $t \in \mathbf{I}, \lambda(x, y, 0)=x$ and $\lambda(x, y, 1)=y$ (such a map is called an equi-connecting map). Using this map, we can define a homotopy $\varphi: Y^{\mathbb{N}} \times Y \times \mathbf{I} \rightarrow Y^{\mathbb{N}}$ as follows: 


$$
\begin{aligned}
\varphi(x, z, 1) & =\left(x_{1}, z, z, z, *, *, \ldots\right), \\
\varphi\left(x, z, 2^{-1}\right) & =\left(x_{1}, x_{2}, z, z, z, *, *, \ldots\right), \\
& \ldots \\
\varphi\left(x, z, 2^{-n}\right) & =\left(x_{1}, \ldots, x_{n}, z, z, z, *, *, \ldots\right), \\
& \ldots \\
\varphi(x, z, 0) & =\left(x_{1}, x_{2}, x_{3}, \ldots\right)=x
\end{aligned}
$$

and for $2^{-n}<t<2^{-n+1}$,

$$
\varphi(x, z, t)=\left(x_{1}, \ldots, x_{n}, \lambda\left(x_{n+1}, z, 2^{n} t-1\right), z, z, \lambda\left(z, *, 2^{n} t-1\right), *, *, \ldots\right) .
$$

Observe that $\varrho(x, \varphi(x, z, t))<t$ for any $t>0$.

Since $A \in \mathcal{E}(X)$, we can take a closed embedding $h: A \hookrightarrow Y=X_{\mathrm{f}}^{\mathbb{N}}$ such that $* \notin h(A)$ and $h(A)$ is closed in $X^{\mathbb{N}}$. Define $g: A \rightarrow \operatorname{Fin}\left(Y^{\mathbb{N}}\right)$ by

$$
g(y)=\{\varphi(x, h(y), \alpha(f(y))) \mid x \in f(y)\} .
$$

It is clear that $g$ is continuous. Since $\varrho(x, \varphi(x, h(y), \alpha(f(y)))<\alpha(f(y))$, it follows that $\varrho_{\mathrm{H}}(f(y), g(y))<\alpha(f(y))$, that is, $g$ is $\alpha$-close to $f$. Note that $\varphi\left(Y^{\mathbb{N}} \times Y \times(0,1]\right) \subset Y_{\mathrm{f}}^{\mathbb{N}}(*)$, which means $g(A) \subset W$. Thus, it remains to prove that $g: A \rightarrow W$ is a $Z$-embedding.

To see that $g$ is injective, let $g(y)=g\left(y^{\prime}\right)$ and fix a point

$$
x=\left(x_{1}, \ldots, x_{n}, *, *, *, \ldots\right) \in g(y)=g\left(y^{\prime}\right)
$$

with $x_{n} \neq *$. Then $x_{n-1}=h(y)=h\left(y^{\prime}\right)$ by the definition of $\varphi$. Since $h$ is an embedding, we have $y=y^{\prime}$.

To see that $g$ is closed, let $a_{i} \in A(i \in \mathbb{N})$ and $G \in \widetilde{W}$ with $g\left(a_{i}\right) \rightarrow G$. We show that $\left(a_{i}\right)_{i \in \mathbb{N}}$ has a convergent subsequence. By taking a subsequence, we may assume that $\alpha\left(f\left(a_{i}\right)\right) \rightarrow t \in \mathbf{I}$. Then $t>0$. Otherwise, $f\left(a_{i}\right) \rightarrow G$ because $\varrho_{\mathrm{H}}\left(f\left(a_{i}\right), g\left(a_{i}\right)\right)<\alpha\left(f\left(a_{i}\right)\right) \rightarrow 0$. Hence, $\alpha\left(f\left(a_{i}\right)\right) \rightarrow \alpha(G)>0$, which is a contradiction. Thus, we can choose $n \in \mathbb{N}$ so that $2^{-n}<t<2^{-n+2}$. Take $z=\left(z_{n}\right)_{n \in \mathbb{N}} \in G \subset\left(X^{\mathbb{N}}\right)^{\mathbb{N}}$. Note that $\varrho\left(z, g\left(a_{i}\right)\right) \rightarrow 0$. For each $i \in \mathbb{N}$, we can choose $x_{i} \in f\left(a_{i}\right)$ so that $\varphi\left(x_{i}, h\left(a_{i}\right), \alpha\left(f\left(a_{i}\right)\right)\right) \rightarrow z$. For sufficiently large $i \in \mathbb{N}, 2^{-n}<\alpha\left(f\left(y_{i}\right)\right)<2^{-n+2}$, in which case

$$
\operatorname{pr}_{n+2} \circ \varphi\left(x_{i}, h\left(a_{i}\right), \alpha\left(f\left(a_{i}\right)\right)\right)=h\left(a_{i}\right),
$$

where $\operatorname{pr}_{n}: Y^{\mathbb{N}} \rightarrow Y$ is the projection onto the $n$th factor. Therefore, $h\left(a_{i}\right) \rightarrow$ $z_{n+2} \in X^{\mathbb{N}}$. Since $h$ is a closed embedding of $A$ not only into $Y$ but also into $X^{N}$, it follows that $\left(a_{i}\right)_{i \in \mathbb{N}}$ is convergent in $A$. Thus, $g: A \rightarrow W$ is closed. Moreover, $g(A)$ is a closed subset of $\widetilde{W}$.

Now, we shall show that $g(A)$ is a $Z$-set in $W$. Let $Y_{\mathrm{f}}^{\mathbb{N}}\left(*^{\prime}\right)$ be the weak product of $Y$ with a base point $*^{\prime}$ different from $*$. Suppose $\mathcal{V}$ is an open cover of $W$. Choose a collection $\widetilde{\mathcal{V}}_{1}$ of open sets of $\operatorname{Fin}\left(\left(X^{\mathbb{N}}\right)^{\mathbb{N}}\right)$ so that $\mathcal{V}=$ $\left\{U \cap \operatorname{Fin}\left(Y_{\mathrm{f}}^{\mathbb{N}}(*)\right) \mid U \in \widetilde{\mathcal{V}}_{1}\right\}$ and $\widetilde{V}=\bigcup \widetilde{\mathcal{V}}_{1} \subset \widetilde{W}$. Let $\widetilde{\mathcal{V}}_{2}$ be an open cover 
of $V$ which is a star-refinement of $\widetilde{\mathcal{V}}_{1}$. Since $\operatorname{Fin}\left(Y_{\mathrm{f}}^{\mathbb{N}}\left(*^{\prime}\right)\right)$ is homotopy dense in $\operatorname{Fin}\left(\left(X^{\mathbb{N}}\right)^{\mathbb{N}}\right)$, there exists a map $i_{1}: \widetilde{V} \rightarrow \widetilde{V} \cap \operatorname{Fin}\left(Y_{\mathrm{f}}^{\mathbb{N}}\left(*^{\prime}\right)\right)$ such that $i_{1}$ is $\widetilde{\mathcal{V}}_{2}$-close to $\mathrm{id}_{\widetilde{V}}$. Recall that $g(A)$ is closed in $\widetilde{V}$. Hence, $\widetilde{V} \backslash g(A)$ is open in $\operatorname{Fin}\left(\left(X^{\mathbb{N}}\right)^{\mathbb{N}}\right)$. Thus, we can find a map

$$
i_{2}: \widetilde{V} \backslash g(A) \rightarrow(\widetilde{V} \backslash g(A)) \cap \operatorname{Fin}\left(Y_{\mathrm{f}}^{\mathbb{N}}(*)\right)
$$

such that $i_{2}$ is $\widetilde{\mathcal{V}}_{2}$-close to $\mathrm{id}_{\widetilde{V}}$. Observe that

$$
g(A) \subset \operatorname{Fin}\left(Y_{\mathrm{f}}^{\mathbb{N}}(*)\right) \quad \text { and } \quad \operatorname{Fin}\left(Y_{\mathrm{f}}^{\mathbb{N}}(*)\right) \cap \operatorname{Fin}\left(Y_{\mathrm{f}}^{\mathbb{N}}\left(*^{\prime}\right)\right)=\emptyset .
$$

Then we have $i_{1}(\widetilde{V}) \subset \widetilde{V} \backslash g(A)$. The $\operatorname{map} i=i_{2} \circ i_{1}: W \rightarrow W$ is $\mathcal{V}$-close to $\operatorname{id}_{W}$ and $i(W) \cap g(A)=\emptyset$. Therefore, $g(A)$ is a $Z$-set in $W$.

By replacing $Y=X_{\mathrm{f}}^{\mathbb{N}}$ with $Y=X^{\mathbb{N}}$ in the proof above, we can also show the following proposition:

Proposition 3.2. Let $X$ be a non-degenerate $A R$ and $W$ an open set in $\operatorname{Fin}\left(X^{\mathbb{N}}\right)$ or $\operatorname{Fin}^{k}\left(X^{\mathbb{N}}\right)$ for some $k \in \mathbb{N}$. Then $W$ is universal for $\mathcal{E}(X)$.

By the same proof as for Proposition 2.2 of [1], we can obtain the following non-separable version:

Proposition 3.3. Let $\mathcal{C}$ be an open and closed hereditary topological class. If each open subset of an $A N R X$ is $\mathcal{C}$-universal and every $Z$-set in $X$ is a strong $Z$-set, then $X$ is strongly $\mathcal{C}$-universal.

Due to Proposition 2.4 in [14], when $\mathcal{C}=\mathfrak{M}_{1}(\tau)$ in the above, it is not necessary to assume that every $Z$-set in $X$ is a strong $Z$-set. Thus, we have the following generalization of Proposition 7.3 of [19]:

Corollary 3.4. Let $X$ be a non-degenerate AR such that $\mathfrak{M}_{1}(\tau) \subset$ $\mathcal{E}(X)$ and let $k \in \mathbb{N}$. Then $\operatorname{Fin}\left(X^{\mathbb{N}}\right), \operatorname{Fin}\left(X_{\mathrm{f}}^{\mathbb{N}}\right), \operatorname{Fin}^{k}\left(X^{\mathbb{N}}\right)$ and $\operatorname{Fin}^{k}\left(X_{\mathrm{f}}^{\mathbb{N}}\right)$ are strongly $\mathfrak{M}_{1}(\tau)$-universal.

By Toruńczyk's characterization of Hilbert spaces [17] (cf. [18]), Theorem 2.4 and Corollary 3.4 imply the following non-separable version of Corollary 2.4 of [9]:

TheOREM 3.5. For each $k \in \mathbb{N}$, the hyperspace $\operatorname{Fin}^{k}\left(\ell_{2}(\tau)\right)$ of the Hilbert space $\ell_{2}(\tau)$ with weight $\tau$ is homeomorphic to $\ell_{2}(\tau)$.

Remark 1. Due to Proposition 6.1 of [19], $\operatorname{Fin}(X)$ is a strong $Z$-space for every normed linear space $X$ with $\operatorname{dim} X \geq 1$. As a combination of Theorems 2.1, 2.4 and Corollary 3.4, we have the main result of [19], that is,

THEOREM 3.6. The hyperspace Fin $\left(\ell_{2}(\tau)\right)$ of the Hilbert space $\ell_{2}(\tau)$ with weight $\tau$ is homeomorphic to $\ell_{2}(\tau) \times \ell_{2}^{\mathrm{f}}$. 


\section{4. $Z$-sets in $\operatorname{Fin}(X)$}

Lemma 4.1. Let $X$ be an $A N R$ and $A$ a $Z$-set in $X$. Then $\operatorname{Fin}(A)$ is a $Z$-set in $\operatorname{Fin}(X)$, and $\operatorname{Fin}^{k}(A)$ is a $Z$-set in $\operatorname{Fin}^{k}(X)$ for any $k \in \mathbb{N}$. Thus, if $X$ is a $Z_{\sigma}$-space then $\operatorname{Fin}(A)$ and $\operatorname{Fin}^{k}(A)$ are also $Z_{\sigma}$-spaces.

Proof. We deal with the case of $\operatorname{Fin}(A)$. Since $X$ is an ANR, $X \backslash A$ is homotopy dense in $X$, hence $\operatorname{Fin}(X \backslash A)$ is homotopy dense in $\operatorname{Fin}(X)$. Since $\operatorname{Fin}(A) \subset \operatorname{Fin}(X) \backslash \operatorname{Fin}(X \backslash A)$, it follows that $\operatorname{Fin}(A)$ is a $Z$-set in $\operatorname{Fin}(X)$. It can be similarly shown that $\operatorname{Fin}^{k}(A)$ is a $Z$-set in $\operatorname{Fin}^{k}(X)$ for any $k \in \mathbb{N}$.

Note that every $Z$-set in $\ell_{2}(\tau)$ is a strong $Z$-set [7]. Since $\ell_{2}(\tau) \times \ell_{2}^{\mathrm{f}}$ is homotopy dense in $\ell_{2}(\tau) \times \ell_{2} \approx \ell_{2}(\tau)$, every $Z$-set in $\ell_{2}(\tau) \times \ell_{2}^{\mathrm{f}}$ is a strong $Z$-set by Lemma 2.2 of [8].

Proposition 4.2. Let $X$ be a non-degenerate $A R$. In the spaces $\operatorname{Fin}\left(X_{\mathrm{f}}^{\mathbb{N}}\right)$ and $\operatorname{Fin}^{k}\left(X_{\mathrm{f}}^{\mathbb{N}}\right), k \in \mathbb{N}$, every $Z$-set is a strong $Z$-set. Thus, $\operatorname{Fin}\left(X_{\mathrm{f}}^{\mathbb{N}}\right)$ and $\operatorname{Fin}^{k}\left(X_{\mathrm{f}}^{\mathbb{N}}\right)$ are strong $Z_{\sigma}$-spaces.

Proof. We may assume that $X_{\mathrm{f}}^{\mathbb{N}}$ can be embedded into Hilbert space as a homotopy dense subset. Indeed, $X$ can be embedded into a completely metrizable $\mathrm{AR} \widetilde{X}$ as a homotopy dense subset [12]. Hence, $X_{\mathrm{f}}^{\mathbb{N}}$ is homotopy dense in $\widetilde{X}^{\mathbb{N}}$ which is homeomorphic to $\ell_{2}(\tau)$ [17]. Thus, $\operatorname{Fin}\left(X_{\mathrm{f}}^{\mathbb{N}}\right)$ and $\operatorname{Fin}^{k}\left(X_{\mathrm{f}}^{\mathbb{N}}\right)$ are homotopy dense subsets of $\operatorname{Fin}\left(\ell_{2}(\tau)\right)$ and $\operatorname{Fin}^{k}\left(\ell_{2}(\tau)\right)$, respectively. Since $\operatorname{Fin}\left(\ell_{2}(\tau)\right) \approx \ell_{2}(\tau) \times \ell_{2}^{\mathrm{f}}$ and $\operatorname{Fin}^{k}\left(\ell_{2}(\tau)\right) \approx \ell_{2}(\tau)$ (Theorems 3.6 and 3.5), it follows from Lemma 2.2 of [8] that every $Z$-set in $\operatorname{Fin}(X)$ is a strong $Z$-set. Since $X_{\mathrm{f}}^{\mathbb{N}}$ is a $Z_{\sigma^{-}}$space, $\operatorname{Fin}\left(X_{\mathrm{f}}^{\mathbb{N}}\right)$ and $\operatorname{Fin}^{k}\left(X_{\mathrm{f}}^{\mathbb{N}}\right)$ are $Z_{\sigma^{-}}$spaces. Thus, they are strong $Z_{\sigma}$-spaces.

REMARK 2. It can also be shown that $\operatorname{Fin}\left(X^{\mathbb{N}}\right)$ is a strong $Z_{\sigma^{-}}$space if $X$ is a non-degenerate AR. Indeed, $\operatorname{Fin}\left(X^{\mathbb{N}}\right)$ can also be embedded into the strong $Z_{\sigma^{-}}$space $\operatorname{Fin}\left(\ell_{2}(\tau)\right) \approx \ell_{2}(\tau) \times \ell_{2}^{\mathrm{f}}$ (Theorem 3.6). Since every AR

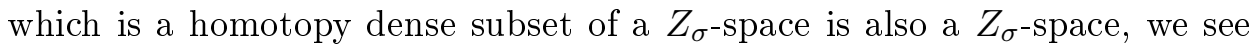
that $\operatorname{Fin}\left(X^{\mathbb{N}}\right)$ is a $Z_{\sigma^{-} \text {-space. }}$

5. Absolute Borel classes. Let $d$ be an admissible metric for $X$. Then the Vietoris topology on $\operatorname{Fin}(X)$ is induced by the Hausdorff metric

$$
d_{\mathrm{H}}(A, B)=\max \left\{\sup _{a \in A} d(a, B), \sup _{b \in B} d(b, A)\right\},
$$

where $d(a, A)=\inf _{y \in A} d(x, y)$. For each $k \in \mathbb{N}$, let $\varrho$ be the metric for $X^{k}$ defined as follows:

$$
\varrho(x, y)=\max _{i \leq k} d\left(x_{i}, y_{i}\right) .
$$

Let $q_{k}: X^{k} \rightarrow \operatorname{Fin}^{k}(X)$ be the natural surjection defined by

$$
q_{k}\left(\left(x_{1}, \ldots, x_{k}\right)\right)=\left\{x_{1}, \ldots, x_{k}\right\} .
$$


Then it is clear that

$$
d_{\mathrm{H}}\left(q_{k}(x), q_{k}(y)\right) \leq \varrho(x, y) \quad \text { for any } x, y \in X^{k} .
$$

This means that $q_{k}$ is uniformly continuous. Note that $\operatorname{card} q_{k}^{-1}(A) \leq k ! \quad$ for each $A \in \operatorname{Fin}^{k}(X)$.

LEMMA 5.1. The map $q_{k}$ is perfect.

Proof. It suffices to show that a sequence $\left(x_{n}\right)_{n \in \mathbb{N}}$ in $X^{k}$ has a convergent subsequence if $\left(q_{k}\left(x_{n}\right)\right)_{n \in \mathbb{N}}$ is convergent in $\operatorname{Fin}^{k}(X)$. Let $q_{k}\left(x_{n}\right)$ be convergent to $A \in \operatorname{Fin}^{k}(X)$. For each $j \leq k$,

$$
d\left(\operatorname{pr}_{j}\left(x_{n}\right), A\right) \leq d_{\mathrm{H}}\left(q_{k}\left(x_{n}\right), A\right) \rightarrow 0 \quad(n \rightarrow \infty),
$$

where $\operatorname{pr}_{j}: X^{k} \rightarrow X$ is the projection onto the $j$ th factor. Since $A$ is finite, any subsequence of $\left(\operatorname{pr}_{j}\left(x_{n}\right)\right)_{n \in \mathbb{N}}$ has a convergent subsequence. Then it is easy to find a subsequence $\left(x_{n_{i}}\right)_{i \in \mathbb{N}}$ such that $\left(\operatorname{pr}_{j}\left(x_{n_{i}}\right)\right)_{i \in \mathbb{N}}$ is convergent in $X$ for every $j \leq k$, which means that $\left(x_{n_{i}}\right)_{i \in \mathbb{N}}$ is convergent in $X^{k}$.

Proposition 5.2. For a $\sigma$-locally compact metric space $X, \operatorname{Fin}(X)$ is also $\sigma$-locally compact, i.e., $X \in \mathfrak{a}_{1}(\tau) \Rightarrow \operatorname{Fin}(X) \in \mathfrak{a}_{1}(\tau)$.

Proof. Let $X=\bigcup_{n \in \mathbb{N}} X_{n}$, where $X_{n}$ is a locally compact subset of $X$ with $X_{n} \subset X_{n+1}$. Since the perfect image of a locally compact space is also locally compact ([4, Theorem 3.7.12]), it follows from Lemma 5.1 that $\operatorname{Fin}^{k}\left(X_{k}\right)$ is locally compact. Then $\operatorname{Fin}(X)=\bigcup_{k \in \mathbb{N}} \operatorname{Fin}^{k}\left(X_{k}\right)$ is $\sigma$-locally compact.

Note that if $f: X \rightarrow Y$ is a closed map and $0<\operatorname{card} f^{-1}(y) \leq k(<\infty)$ for every $y \in Y$ then $\operatorname{dim} Y \leq \operatorname{dim} X+k-1$ [5, Theorem 3.3.7]. Then, by the same proof as for Proposition 5.2 above, we have the following:

Proposition 5.3. $X \in \mathfrak{a}_{1}^{\omega}(\tau) \Rightarrow \operatorname{Fin}(X) \in \mathfrak{a}_{1}^{\omega}(\tau)$.

By Proposition 5.1 of [19], if $X$ is completely metrizable then $\operatorname{Fin}(X)$ is $\sigma$-completely metrizable, that is,

$$
X \in \mathfrak{M}_{1}(\tau) \Rightarrow \operatorname{Fin}(X) \in \mathfrak{M}_{1}(\tau)_{\sigma} \subset \mathfrak{a}_{2}(\tau) .
$$

We also have the following:

$$
X \in \mathfrak{M}_{1}(\tau)_{\sigma} \Rightarrow \operatorname{Fin}(X) \in \mathfrak{M}_{1}(\tau)_{\sigma} .
$$

Proposition 5.4. For each countable ordinal $\alpha \geq 2$,

$$
\begin{gathered}
X \in \mathfrak{a}_{\alpha}(\tau) \Rightarrow \operatorname{Fin}(X) \in \mathfrak{a}_{\alpha}(\tau), \\
X \in \mathfrak{M}_{\alpha}(\tau) \Rightarrow \operatorname{Fin}(X) \in \mathfrak{M}_{\alpha}(\tau) .
\end{gathered}
$$

Proof. We handle the cases of $X \in \mathfrak{a}_{2}(\tau)$ and $X \in \mathfrak{M}_{2}(\tau)$. Then the result can be obtained by transfinite induction. 
If $X \in \mathfrak{a}_{2}(\tau)$, let $X=\bigcup_{i \in \mathbb{N}} X_{i}$, where each $X_{i}$ is completely metrizable. Without loss of generality, we may assume $X_{i} \subset X_{i+1}$ for each $i \in \mathbb{N}$; then $\operatorname{Fin}(X)=\bigcup_{i \in \mathbb{N}} \operatorname{Fin}\left(X_{i}\right)$. Since each $\operatorname{Fin}\left(X_{i}\right)$ is $\sigma$-completely metrizable, $\operatorname{Fin}(X)$ is also $\sigma$-completely metrizable. This means $\operatorname{Fin}(X) \in \mathfrak{a}_{2}(\tau)$.

If $X \in \mathfrak{M}_{2}(\tau)$, let $\widetilde{X}$ be the completion of $X$. Since $X$ is $F_{\sigma \delta}$ in $\widetilde{X}$, $\operatorname{Fin}(X)$ is also $F_{\sigma \delta}$ in $\operatorname{Fin}(\widetilde{X})$. By Proposition 5.1 of [19], $\operatorname{Fin}(\widetilde{X})$ is $F_{\sigma}$ in the completely metrizable space $\operatorname{Cld}_{\mathrm{H}}(\widetilde{X})$. This means $\operatorname{Fin}(X)$ is $F_{\sigma \delta}$ in $\operatorname{Cld}_{\mathrm{H}}(\widetilde{X})$. Thus, $\operatorname{Fin}(X) \in \mathfrak{M}_{2}(\tau)$.

Remark 3. For each $k \in \mathbb{N}, \operatorname{Fin}^{k}(X)$ is closed in $\operatorname{Fin}(X)$. For the spaces $\operatorname{Fin}^{k}(X), k \in \mathbb{N}$, we have the same results as for $\operatorname{Fin}(X)$ above.

\section{Proof of the Main Theorem. First, we prove the following:}

THEOREM 6.1. Let $\mathcal{C}$ be an open and closed hereditary, additive, productive and topological class of spaces such that $\mathbf{I}^{n} \times D(\tau) \in \mathcal{C}$ for each $n \in \mathbb{N}$. Suppose that there exists a $\mathcal{C}$-absorbing set $E$ in $\ell_{2}(\tau)$. Then $\operatorname{Fin}(E)$ and $\operatorname{Fin}^{k}(E), k \in \mathbb{N}$, are strongly $\mathcal{C}$-universal.

Proof. By the $\mathcal{C}$-universality of $E$, we have $\mathcal{C} \subset \mathcal{E}(E)$. Since $\operatorname{Fin}\left(E_{\mathrm{f}}^{\mathbb{N}}\right)$ and $\operatorname{Fin}^{k}\left(E_{\mathrm{f}}^{\mathbb{N}}\right)$ are AR's by Theorem 2.4 and every $Z$-set is a strong $Z$-set in these spaces, it follows from Propositions 3.1 and 3.3 that $\operatorname{Fin}\left(E_{\mathrm{f}}^{\mathbb{N}}\right)$ and $\operatorname{Fin}^{k}\left(E_{\mathrm{f}}^{\mathbb{N}}\right)$ are strongly $\mathcal{C}$-universal. On the other hand, $E_{\mathrm{f}}^{\mathbb{N}} \approx E$ by Proposition 2.3 , hence $\operatorname{Fin}(E) \approx \operatorname{Fin}\left(E_{\mathrm{f}}^{\mathbb{N}}\right)$. Thus, we have the result.

Now, we shall prove the main theorem.

THEOREM 6.2. Suppose that $E$ is homeomorphic to $\ell_{2}^{\mathrm{f}}(\tau), \Lambda_{\alpha}(\tau)$ or $\Omega_{\alpha}(\tau)$, where $\alpha \geq 1$ is a countable ordinal. Then the hyperspaces $\operatorname{Fin}(E)$ and $\operatorname{Fin}^{k}(E), k \in \mathbb{N}$, are homeomorphic to $E$.

Proof. First, note that $E$ is strongly universal for the class $\mathcal{C}=\mathcal{E}(E)$ and $E \in \mathcal{C}_{\sigma}=\mathcal{C}$. In $\S 5$, we have shown that $\operatorname{Fin}(E), \operatorname{Fin}^{k}(E) \in \mathcal{C}$. These spaces are strong $Z_{\sigma}$-spaces by Proposition 4.2 and are strongly $\mathcal{C}$-universal by Theorem 6.1 . Thus, $\operatorname{Fin}(E) \approx \operatorname{Fin}^{k}(E) \approx E$ by Theorem 2.1 .

Since every connected $E$-manifold $X$ can be embedded into $E$ as an open set by Theorem 2.2, $\operatorname{Fin}(X)$ and $\operatorname{Fin}^{k}(X)$ can also be embedded into $\operatorname{Fin}(E)$ and $\operatorname{Fin}^{k}(E)$ as open sets, respectively. Since $X$ is connected, $\operatorname{Fin}(X)$ is an AR (cf. Proposition 3.1 of [19]) and each $\operatorname{Fin}^{k}(X)$ is connected. Hence, we have the following theorem.

Theorem 6.3. Suppose that $E$ is homeomorphic to $\ell_{2}^{\mathrm{f}}(\tau), \Lambda_{\alpha}(\tau)$ or $\Omega_{\alpha}(\tau)$, where $\alpha \geq 1$ is a countable ordinal. Let $X$ be a connected E-manifold. Then $\operatorname{Fin}(X)$ is homeomorphic to $E$ and each $\operatorname{Fin}^{k}(X)$ is a connected $E$ manifold. 


\section{References}

[1] M. Bestvina and J. Mogilski, Characterizing certain incomplete infinite-dimensional absolute retracts, Michigan Math. J. 33 (1986), 291-313.

[2] D. Curtis, Hyperspaces of finite subsets as boundary sets, Topology Appl. 22 (1986), 97-107.

[3] D. Curtis and Nguyen To Nhu, Hyperspaces of finite subsets which are homeomorphic to $\aleph_{0}$-dimensional linear metric spaces, ibid. 19 (1985), 251-260.

[4] R. Engelking, General Topology, rev. ed., Sigma Ser. Pure Math. 6, Heldermann, Berlin, 1989.

[5] -, Theory of Dimensions, Finite and Infinite, Sigma Ser. Pure Math. 10, Heldermann, Berlin, 1995.

[6] V. V. Fedorchuk, Covariant functors in the category of compacta, absolute retracts, and Q-manifolds, Uspekhi Mat. Nauk 36 (1981), no. 3, 177-195 (in Russian); English transl.: Russian Math. Surveys 36 (1981), no. 3, 211-233.

[7] D.W. Henderson, Z-sets in ANR's, Trans. Amer. Math. Soc. 213 (1975), 205-216.

[8] K. Mine, Universal spaces of non-separable absolute Borel classes, Tsukuba J. Math., to appear.

[9] Nguyen To Nhu, Investigating the ANR-property of metric spaces, Fund. Math. 124 (1984), 243-254.

[10] - , Hyperspaces of compact sets in metric linear spaces, Topology Appl. 22 (1986), 109-122.

[11] Nguyen To Nhu and K. Sakai, Probability measure functions preserving infinitedimensional spaces, Colloq. Math. 70 (1996), 291-304.

[12] K. Sakai, The completion of metric ANR's and homotopy dense subsets, J. Math. Soc. Japan 52 (2000), 835-846.

[13] K. Sakai and M. Yaguchi, Characterizing manifolds modeled on certain dense subspaces of non-separable Hilbert spaces, Tsukuba J. Math. 27 (2003), 143-159.

[14] - - - Hyperspaces of Banach spaces with the Attouch-Wets topology, Set-Valued Anal. 12 (2004), 329-344.

[15] A. H. Stone, Absolute F $F_{\sigma}$ spaces, Proc. Amer. Math. Soc. 13 (1962), 495-499.

[16] H. Toruńczyk, Concerning locally homotopy negligible sets and characterization of $\ell_{2}$-manifolds, Fund. Math. 101 (1978), 93-110.

[17] —, Characterizing Hilbert space topology, ibid. 111 (1981), 247-262.

[18] —, A correction of two papers concerning Hilbert manifolds, ibid. 125 (1985), 89-93.

[19] M. Yaguchi, Hyperspaces of finite subsets of non-separable Hilbert spaces, Tsukuba J. Math., to appear.

K. Mine, K. Sakai and M. Yaguchi

Institute of Mathematics

University of Tsukuba

Tsukuba, 305-8571 Japan

E-mail: pen@math.tsukuba.ac.jp

sakaiktr@sakura.cc.tsukuba.ac.jp

masato@math.tsukuba.ac.jp

Received May 30, 2005;

received in final form February 20, 2006 